\title{
Energetic particle counterparts for geomagnetic pulsations of Pc1 and IPDP types
}

\author{
T. A. Yahnina ${ }^{1}$, A. G. Yahnin ${ }^{1}$, J. Kangas ${ }^{2}$, J. Manninen ${ }^{2}$, D. S. Evans ${ }^{3}$, A. G. Demekhov ${ }^{4}$, V. Yu. Trakhtengerts ${ }^{4}$, \\ M. F. Thomsen ${ }^{5}$, G. D. Reeves ${ }^{5}$, and B. B. Gvozdevsky ${ }^{1}$ \\ ${ }^{1}$ Polar Geophysical Institute, Apatity, Murmansk region, Russia \\ ${ }^{2}$ Sodankylä Geophysical Observatory, Sodankylä, Finland \\ ${ }^{3}$ NOAA Space Environmental Center, Boulder, Colorado, USA \\ ${ }^{4}$ Institute of Applied Physics, Russian Academy of Sciences, Nizhniy Novgorod, Russia \\ ${ }^{5}$ Los Alamos National Laboratory, Los Alamos, NM, USA
}

Received: 9 December 2002 - Revised: 16 March 2003 - Accepted: 13 May 2003

\begin{abstract}
Using the low-altitude NOAA satellite particle data, we study two kinds of localised variations of energetic proton fluxes at low altitude within the anisotropic zone equatorward of the isotropy boundary. These flux variation types have a common feature, i.e. the presence of precipitating protons measured by the MEPED instrument at energies more than $30 \mathrm{keV}$, but they are distinguished by the fact of the presence or absence of the lower-energy component as measured by the TED detector on board the NOAA satellite. The localised proton precipitating without a lowenergy component occurs mostly in the morning-day sector, during quiet geomagnetic conditions, without substorm injections at geosynchronous orbit, and without any signatures of plasmaspheric plasma expansion to the geosynchronous distance. This precipitation pattern closely correlates with ground-based observations of continuous narrow-band $\mathrm{Pc} 1$ pulsations in the frequency range $0.1-2 \mathrm{~Hz}$ (hereafter Pc1). The precipitation pattern containing the low energy component occurs mostly in the evening sector, under disturbed geomagnetic conditions, and in association with energetic proton injections and significant increases of cold plasma density at geosynchronous orbit. This precipitation pattern is associated with geomagnetic pulsations called Intervals of Pulsations with Diminishing Periods (IPDP), but some minor part of the events is also related to narrow-band Pc1. Both Pc1 and IPDP pulsations are believed to be the electromagnetic ion-cyclotron waves generated by the ion-cyclotron instability in the equatorial plane. These waves scatter energetic protons in pitch angles, so we conclude that the precipitation patterns studied here are the particle counterparts of the ion-cyclotron waves.
\end{abstract}

Key words. Ionosphere (particle precipitation) - Magnetospheric physics (energetic particles, precipitating) - Space plasma physics (wave-particle interactions)

Correspondence to: A. G. Yahnin

(yahnin@pgi.kolasc.net.ru)

\section{Introduction}

Continuous structured narrow-band geomagnetic pulsations, having a frequency within the range of $0.1-5 \mathrm{~Hz}$, termed Pc1, occur in the morning-day sector during quiet geomagnetic conditions, mainly during the recovery of a geomagnetic storm (e.g. Wentworth, 1964; Heacock and Kivinen, 1972). The pulsations called Intervals of Pulsations with Diminishing Periods (IPDP) resemble Pc1, whose frequency increases in time (typically from 0.2 to $1-2 \mathrm{~Hz}$ ). These pulsations occur in the evening sector in association with the expansion phase of a substorm (e.g. Troitskaya, 1961; Hayakawa et al., 1992). In spite of the morphological difference, these two types of the ULF waves are commonly suggested to result from the ion-cyclotron instability in the inner magnetosphere (e.g. Kangas et al., 1998, and references therein). The free energy for the instability is the anisotropy of ringcurrent protons. The instability causes pitch-angle diffusion, which leads to partial filling of the loss cone and, hence, precipitation of energetic particles. The precipitation should modify the ionospheric properties (in particular, the reflection coefficient of Alfvén waves from the ionosphere). This may affect the self-consistent behaviour of the Alfvén ioncyclotron (Pc1) wave packets bouncing between the hemispheres and, hence, the formation of their dynamic spectrum (Belyaev et al., 1984; Trakhtengerts et al., 2000a). Another aspect of these wave-particle interactions is their role in the decay of the ring current due to the loss of particles scattered into the loss cone (e.g. Bespalov et al., 1994; Grafe et al., 1996; Kozyra et al., 1997). The knowledge of parameters of the precipitating particles is very important for verification of the assumed mechanisms. However, since the pioneering theoretical work by Gintzburg (1961) and Cornwall (1965), the problem of detection of the specific proton precipitation related to Pc1 was unsolved until recently. In spite of the importance of the problem, there is only very limited information on the particles directly precipitated by the ion-cyclotron waves. 
Note that it is often difficult to observe the loss-cone filling near the equatorial plane where the loss cone is very small (only a few degrees) and cannot be resolved by a particle detector. To our knowledge, the only observations near the equatorial plane, experimentally evidencing the proton pitchangle diffusion related to the electromagnetic ion-cyclotron (EMIC) waves, are those published very recently by Erlandson and Ukhorskiy (2001). They considered protons with relatively low energies $(E<17 \mathrm{keV})$ and found the behaviour of this population to be consistent with the ion-cyclotron instability.

It is much easier to detect precipitation at low altitudes where the loss cone is larger than the field of view of a typical particle detector. But, to our knowledge, the only previous paper that related a proton precipitation pattern to geomagnetic pulsations in the Pc1 range (namely, to the IPDP pulsations) was the paper by Søraas et al. (1980), who used the energetic-proton data from the ESRO-1 satellite. They observed the bursts of precipitating particles equatorward from the isotropy boundary (the boundary dividing the isotropic precipitation at the poleward side from the trapped population equatorward) during some IPDP events. Only recently, Yahnina et al. (1998, 2000, 2002) found a close relationship between localised precipitation of energetic protons equatorward of isotropy boundary with ground structured Pc1 pulsations ("pearls") using the NOAA low-altitude satellite data and studied this relationship statistically. The localised precipitation bursts were detected mainly in the energy range of $30-80 \mathrm{keV}$, and their spectra had no detectable extension to the lower energy $(<20 \mathrm{keV})$. Yahnina et al. also noted that morphological features of this precipitation type are in agreement with the features of EMIC waves observed in space at the same latitudes. The authors concluded that the localized proton precipitation marks the Pc1 source field lines. Thus, the localised proton precipitation detected within the zone of anisotropic fluxes should carry an information about the EMIC waves source region. This fact stresses the importance of a detailed study of the characteristics of such precipitation.

Some features of the localised enhancements of energetic proton fluxes (not only precipitating, but mainly locally mirroring) have been discussed by Lundblad and Søraas (1978) on the basis of the ESRO-1 satellite data and more recently by Søraas et al. (1999) on the basis of the NOAA-6 satellite data. However, these authors did not correlate these enhancements with Pc1.

As noted by Yahnina et al. $(2000,2002)$, the presence of the precipitating particles (i.e. particles within the loss cone) in the localised flux enhancements means a relatively strong diffusion process in the source region, while the enhancements of the only trapped population may be the result of particle drift from the source region or rather weak diffusion. Below we will try to further investigate the morphology of the localised proton precipitation using the data from NOAA-12 satellite and some other data obtained during the year 1996. In Sect. 2 we briefly describe the data used and criteria for the event selection. Section 3 is devoted to a study of the characteristics of the precipitation of inter- est and their correlations with different related phenomena, including geomagnetic activity indices, plasma parameters at geosynchronous orbit, and geomagnetic pulsations. The results will be discussed in Sect. 4.

\section{Data and event selection}

\subsection{The NOAA satellite instrumentation}

The satellites of the NOAA POES series have the polar circular orbit at altitude of some $800 \mathrm{~km}$. For this study the data from two particle instruments on board the NOAA-12 satellite, which crosses the polar region approximately along the meridian 21:00-09:00 MLT, were used.

The MEPED instrument measures energetic particles with four solid-state detector telescopes, two devoted to observing electrons and two to protons. The NOAA satellites are three-axis stabilised, and one pair of electron and proton detectors view along the Earth-satellite radial vector. At high latitudes $(L>3)$ the detectors viewing along this direction measures particles within the loss cone. The second pair of detectors view perpendicularly to the Earth-satellite vector and observe particles that will magnetically mirror above the atmosphere. Protons are measured in several energy channels. Three lower energy channels of the MEPED instrument (P1, P2, and P3) have nominal energy passbands of 30$80 \mathrm{keV}, 80-250 \mathrm{keV}$, and $250-800 \mathrm{keV}$, respectively. However, it is necessary to note that the solid-state detectors in the proton telescopes suffer radiation damage over time. The impact of this damage is to increase the energy threshold for counting protons from their nominal levels to levels significantly higher. The estimate of the threshold made by comparison with the NOAA-15 satellite data for three-month interval of 1998 showed that after 6.5 years of the NOAA-12 operation, the $30 \mathrm{keV}$ and $80 \mathrm{keV}$ energy thresholds in the $0^{\circ}$ proton detector increased, respectively, to $50-65 \mathrm{keV}$ and $135-165 \mathrm{keV}$ (depending on assumptions). The thresholds in the $90^{\circ}$ telescope increased to $75 \mathrm{keV}$ and $175 \mathrm{keV}$, respectively. Keeping this fact in mind, as well as the uncertainty in the threshold level for 1996, below we will refer to the measurements in different channels as measurements in the P1-P3 channels, thereby avoiding the specific energy values.

For three electron channels of the MEPED instrument the energy thresholds are $>30 \mathrm{keV},>100 \mathrm{keV}$, and $>300 \mathrm{keV}$. The TED instrument measures the total energy flux of particles with energy less than $20 \mathrm{keV}$ by two detectors; both viewing within the loss cone at high latitudes. Details of the NOAA particle measurements can be found in Hill et al. (1985) and Kroehl (1982).

\subsection{Geosynchronous data}

In Sect. 3 we compare the NOAA data with some phenomena observed at geosynchronous orbit. The instruments used for this comparison are the Synchronous Orbit Particle Analyser (SOPA) and Magnetospheric Plasma Analyser (MPA), on board geosynchronous LANL spacecraft (see Belian et al., 


\section{October 1996 NOAA-12}

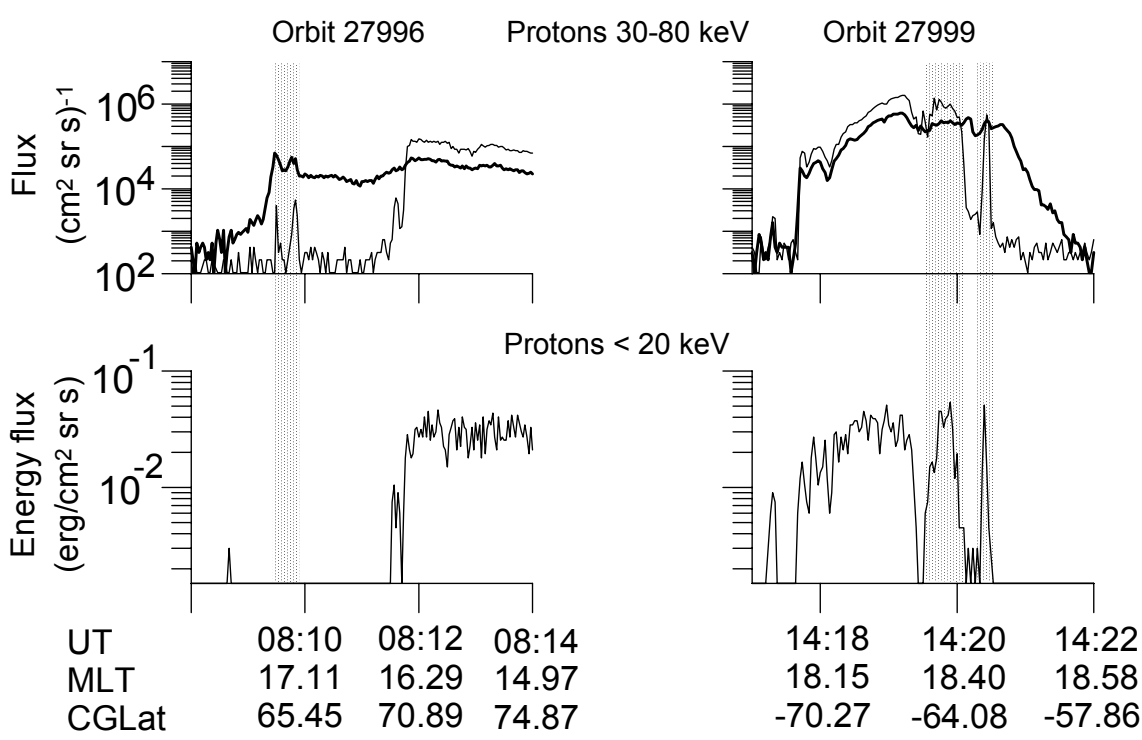

(a)

(b)

Fig. 1. Examples of localized proton flux enhancements detected in the anisotropic zone by the NOAA-12 satellite. Upper panels shows the data from the MEPED instrument measuring the particles with $E>30 \mathrm{keV}$. Thick line presents trapped flux; thin line presents the precipitating flux. Bottom panels show the data from TED instrument (precipitating particles with $E<20 \mathrm{keV}$ ). (a) The flux enhancement, which does not contain the low-energy component (Type 1); (b) The flux enhancement with the low-energy component (Type 2).

1992; Bame et al., 1993). The SOPA instrument measures both electrons and protons with energies $E>50 \mathrm{keV}$; in this paper, the data from five "low energy" channels are used. The MPA instrument measures particles within the energy range from $1 \mathrm{eV}$ to $40 \mathrm{keV}$. In this paper, the density of protons with energy less than $10 \mathrm{eV}$ is used to retrieve the information on the cold plasma distribution along the geosynchronous orbit.

\subsection{Ground-based magnetic data}

The presence of the Pc1/IPDP activity is detected using the data from the search coil magnetometer situated at Sodankylä Geophysical Observatory (SGO), $L=5.2$. Geographic coordinates of SGO are: $67^{\circ} 22^{\prime} \mathrm{N}, 26^{\circ} 38^{\prime} \mathrm{E}$. The instrument is a part of the Finnish search coil magnetometer network. Information about the network can be found on the Internet (http://spaceweb.oulu.fi/projects/pulsations/). Since mid1995, the instrument provides the data in digital form. The data are routinely processed, and daily spectrograms of the geomagnetic field variations in the frequency range 0.1 to $4.0 \mathrm{~Hz}$ are continuously produced. In the present study, the data for whole year 1996 were used for a correlation with the particle precipitation data.

\subsection{Event selection}

Searching through the NOAA-12 data for 1996, we selected localised (about 1 degree in latitude) enhancements of the precipitating energetic proton flux within the anisotropic zone (equatorward of the isotropy boundary). The preliminary selection was made from the MEPED energy channel P1. On the whole, 457 such events were found. The flux enhancements were then divided into two groups.

One group (169 events) contained the localised proton enhancements similar to those studied by Yahnina et al. (2000, 2002). This type of flux enhancement did not contain any measurable energy flux of low-energy $(<20 \mathrm{keV})$ protons as measured by the TED instrument. An example of this type of event is shown in Fig. 1a. At the top panel of Fig. 1a the data from the MEPED instrument are presented. Thick and thin lines show the trapped and precipitating flux intensities, respectively. The regions of isotropic and anisotropic fluxes are clearly seen in the data. (Note the dominance of precipitating fluxes over the trapped fluxes within the isotropy region. This is due to the difference in the energy thresholds for $0^{\circ}$ and $90^{\circ}$ detectors as mentioned in Sect. 2.1.) Within the anisotropic zone, a sharp enhancement of the precipitating flux, together with the prominent variation of the trapped flux intensity, is seen at 08:09:32 UT. It is just this type of flux enhancement that is of interest for us. The bottom panel of Fig. 1a shows precipitating protons detected by the TED instrument. The behaviour of the low-energy proton precipitation at higher latitudes is similar to that of energetic protons within the isotropy region. This agrees with the fact that protons with energy of a few tens of $\mathrm{keV}$ are a substantial part of the plasma sheet population (Christon et al., 1988, 1989). The energy flux of the low-energy protons starts to diminish 

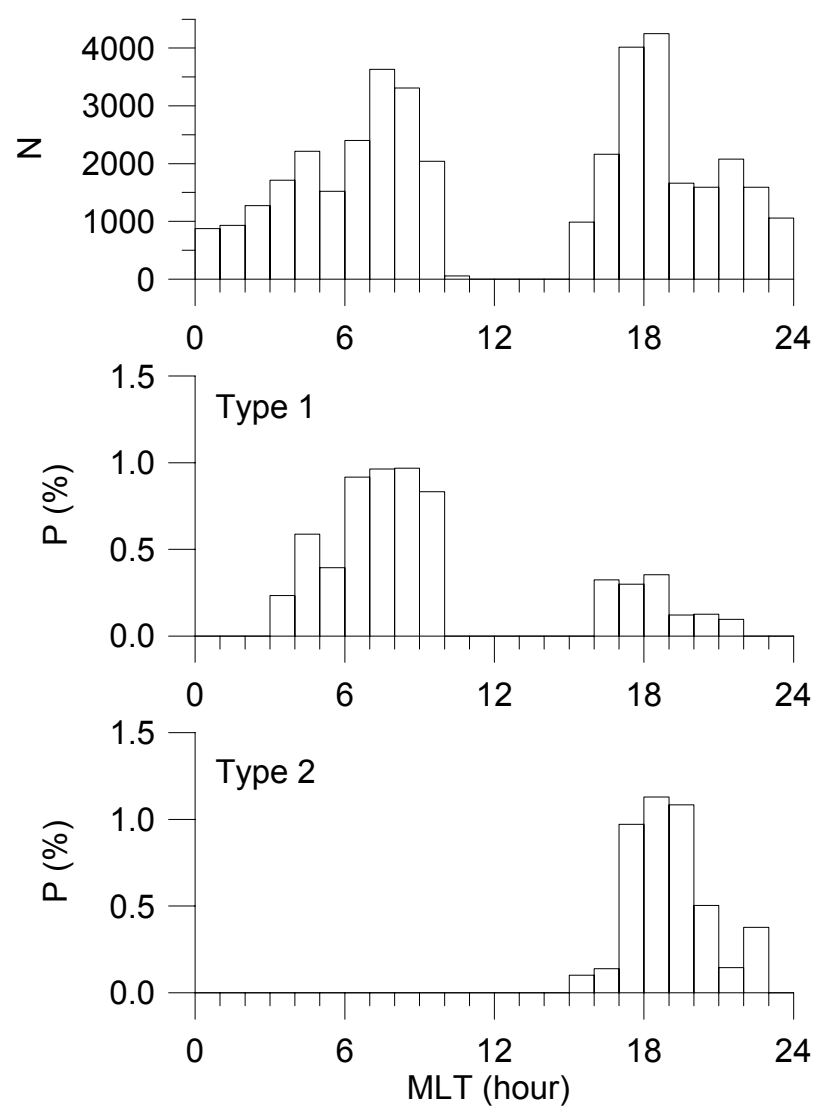

Fig. 2. Observational statistics for localized proton precipitations as a function of MLT. Upper panel: number of NOAA-12 passes in 1-h MLT intervals for 1996. Middle panel: Occurrence rate of the Type 1 events. Bottom panel: Occurrence rate for the Type 2 events.

close to (slightly poleward of) the energetic proton isotropy boundary (IB), in agreement with results by Gvozdevsky and Sergeev (1997) (see also Newell et al., 1998, who used the start of the flux reduction at the equatorial edge of the low energetic proton precipitation as a proxy for IB). The lowenergy precipitation is completely absent at the latitude of the high-energy proton enhancement within the anisotropic zone. Hereafter, we will refer to this type of proton flux enhancement as the event of Type 1.

Another group of the proton enhancements (288 events), which we will refer to as Type 2, consist of the localised enhancements like those shown in the upper panel of Fig. 1b. In fact, there are two localised enhancements of energetic protons equatorward of the isotropy boundary: one of them is clearly seen at 14:20:26 UT, and another one, at 14:19:50 UT, that overlaps the isotropic precipitation. These enhancements differ from those shown in Fig. 1a by the presence of bursts of low-energy protons seen in the bottom panel. The lowenergy burst at 14:19:50 UT allows us to distinguish the corresponding enhancement in the $\mathrm{P} 1$ channel from the isotropic precipitation zone. Thus, the difference between the two types of the localised enhancements, by our definition, is only in the absence or presence of the lower-energy extension in the energetic proton spectra during the precipitating flux enhancement. This difference can seem to be insignificant. However, as we show below, that difference correlates with much clearer differences in certain other characteristics (i.e. in properties of accompanying ULF emissions).

\section{Properties of the Type 1 and 2 localised enhancements of proton flux}

\subsection{Occurrence rate, dependence on MLT}

The localised proton bursts of both types were observed at magnetic latitudes $58-70^{\circ}$. Figure 2 shows the number of NOAA-12 passes at any MLT sector within this latitudinal interval. Both crossings in the Northern and Southern Hemispheres are counted. Due to the orbit peculiarities, the satellite passes did not cover the local time range of 10:0015:00 MLT. The ratio of the number of passes when the proton enhancements were detected to the number of passes within the given MLT hour determines the occurrence rate of the observations for this MLT. Thus, the middle and bottom panels show the occurrence rates of the enhancements of Types 1 and 2, respectively. The Type 1 events have the maximum of occurrence in the morning. (It is possible, however, that the maximum extends to the dayside where there are no observations.) The Type 2 events occur mainly in the evening sector.

The occurrence probability of these events is rather small (only $\sim 0.01$ and 0.02 in the maximum of the MLT distribution for the Types 1 and 2, respectively).

\subsection{Intensity of the precipitation, isotropization, energy} spectra, and relation to the electron precipitation

Figure 3a shows a normalised distribution of the events of both types as a function of the flux intensity in the P1 channel of MEPED. This figure represents some characteristics of the distributions (median values, first and third quartiles). It is clearly seen that Type 2 events have much more intense flux in comparison to the Type 1 events. About $70 \%$ of the Type 2 events have the flux intensity above $10^{5}\left(\mathrm{~cm}^{2} \mathrm{~s} \mathrm{sr}\right)^{-1}$. At the same time some $70 \%$ of the Type 1 events have the intensity below this value. An important characteristic of a pitch-angle diffusion process is the isotropization of the pitch-angle distribution. To quantify the isotropization, we use the ratio of precipitating and trapped fluxes at the NOAA satellite altitude, which corresponds to the ratio of the fluxes within and just outside the loss cone in the equatorial plane. Figure $3 \mathrm{~b}$ demonstrates the normalised distribution of the Type 1 and 2 events as a function of the isotropization degree. The isotropization for Type 2 events is much higher. This is characterised by the fact that some $70 \%$ of the Type 2 events have the isotropization parameter higher than 1 , while for about $70 \%$ of the Type 1 events this parameter is below 1. (The dominance of precipitating flux over trapped fluxes is the result of different thresholds of $0^{\circ}$ and $90^{\circ}$ proton sensors, as mentioned in the Introduction.) Finally, Fig. $3 \mathrm{c}$ shows the 
isotropization parameter for the two precipitation types as a function of the precipitating flux.

To characterise the hardness of the precipitating particle spectra we considered the ratio of the flux intensity in channels $\mathrm{P} 2$ and P1. This ratio is the same for both types of precipitation in a wide range of the precipitating flux and is equal to about 0.1 . Thus, both Type 1 and Type 2 spectra have a maximum in the energy range of the MEPED P1 channel (several tens of $\mathrm{keV}$, see Introduction) and the flux decrease with increasing energy is similar for both types of events. However, the spectra of the Type 2 events are wider, and this relates, according to our definition, to the low energy extension of the spectra.

The Type 2 events are very often ( $82 \%$ of events) accompanied by localised precipitation of energetic electrons with energies $>30 \mathrm{keV}$. This association is much less typical for the Type 1 events (44\% of the cases). Here we omit the corresponding figures for brevity; the examples of this relationship can be found in Yahnina et al. (2000, 2002).

\subsection{Relation to geomagnetic activity and substorms}

The events of Types 1 and 2 are very different in terms of their relation to the geomagnetic activity. Figure 4a demonstrates this fact by correlation with the $K_{p}$ index. The two upper panels show the numbers of Type 1 and 2 events observed in 1996 as functions of $K_{p}$. The middle panel represents the distribution of $K_{p}$ indices for the entire year 1996. Finally, the two lower panels show the occurrence rates of the Type 1 and 2 events within every $K_{p}$ interval (obtained by dividing the numbers in the upper panels by the corresponding number in the middle panel). The Type 1 events exhibit a clear tendency to be observed more often when $K_{p}$ is smaller. The dependence of the Type 2 occurrence on $K_{p}$ is opposite.

The dependence on the $D_{s t}$-index shown in Fig. $4 \mathrm{~b}$ confirms this tendency. This plot is constructed similar to Fig. 4a. Again, the occurrence rates for the two types of precipitation demonstrate the opposite dependences on $D_{s t}$. Note that for the Type 2 events the extremely high values of the occurrence rate for bins $-100<D_{s t}<-80 \mathrm{nT}(\sim 30 \%)$ and $20<D_{s t}<40 \mathrm{nT}(\sim 5 \%)$ are doubtful, due to poor statistics. There were only two events in the $-100 \mathrm{nT}$ to $-80 \mathrm{nT}$ bin (both occurred during the magnetic storm of 13 January, the biggest storm in 1996), and only one event in the $-40 \mathrm{nT}$ to $-20 \mathrm{nT}$ bin.

Another important characteristic of the precipitation events is their relation to substorms. The appearance of energetic particle injections at geosynchronous orbit is often used as an indicator of substorm activity. We checked the available SOPA data from the LANL spacecraft and found that the Type 2 events were always preceded by the injection of the energetic protons. An example of such a relationship is shown in Fig. 5 for the case observed at 11:05:30 UT on 4 November. The NOAA data for this case are shown in Fig. 5a. Figure 5b presents the LANL spacecraft data. Just before the time of the Type 2 event detection the LANL 1995-084 and 1990-095 spacecraft situated in the evening
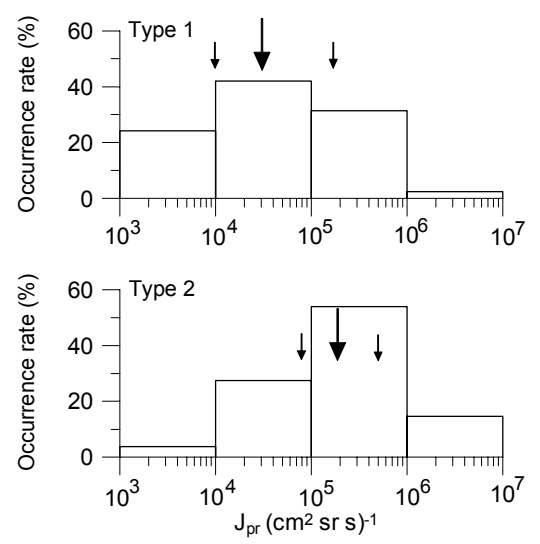

(a)
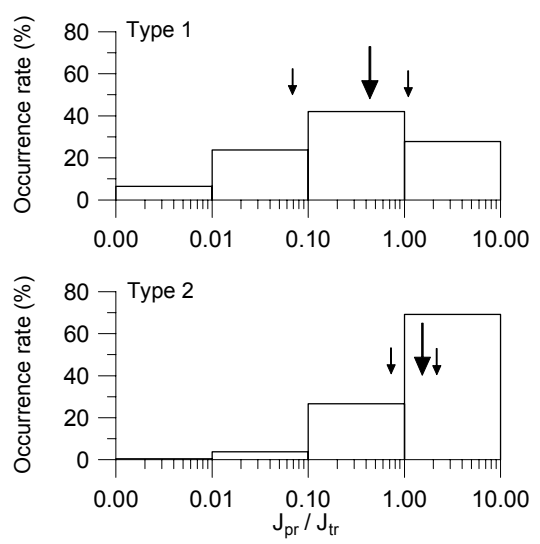

(b)

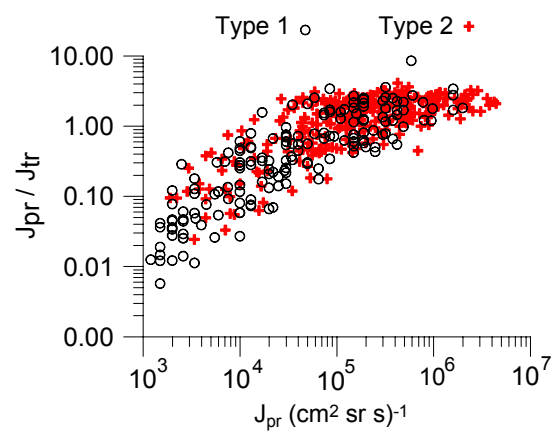

(c)

Fig. 3. Features of the localized proton precipitation. (a) Normalized distribution of the events as a function of the precipitating particle flux. Median value of the flux (large arrow), as well as first and third quartiles (small arrows) are indicated. (b) Normalized distribution of the events as a function of the $J_{\text {prec }} / J_{\text {trap }}$ ratio. Median value of the izotropization parameter, as well as first and third quartiles are shown as above. (c) Dependence of isotropization parameter $\left(J_{\text {prec }} / J_{\text {trap }}\right)$ on the precipitating flux for events of Type 1 (circles) and Type 2 (crosses).

sector registered the energetic proton injection. Note that the MLT of LANL 084 was very close to the MLT of the NOAA12 pass.

A comparison of a Type 1 event with the LANL data is shown in Fig. 6. The precipitation was detected on 1 November at about 05:40:42 UT at 9 MLT (Fig. 6a). Close to this time the LANL 1991-080 spacecraft was in the same MLT 

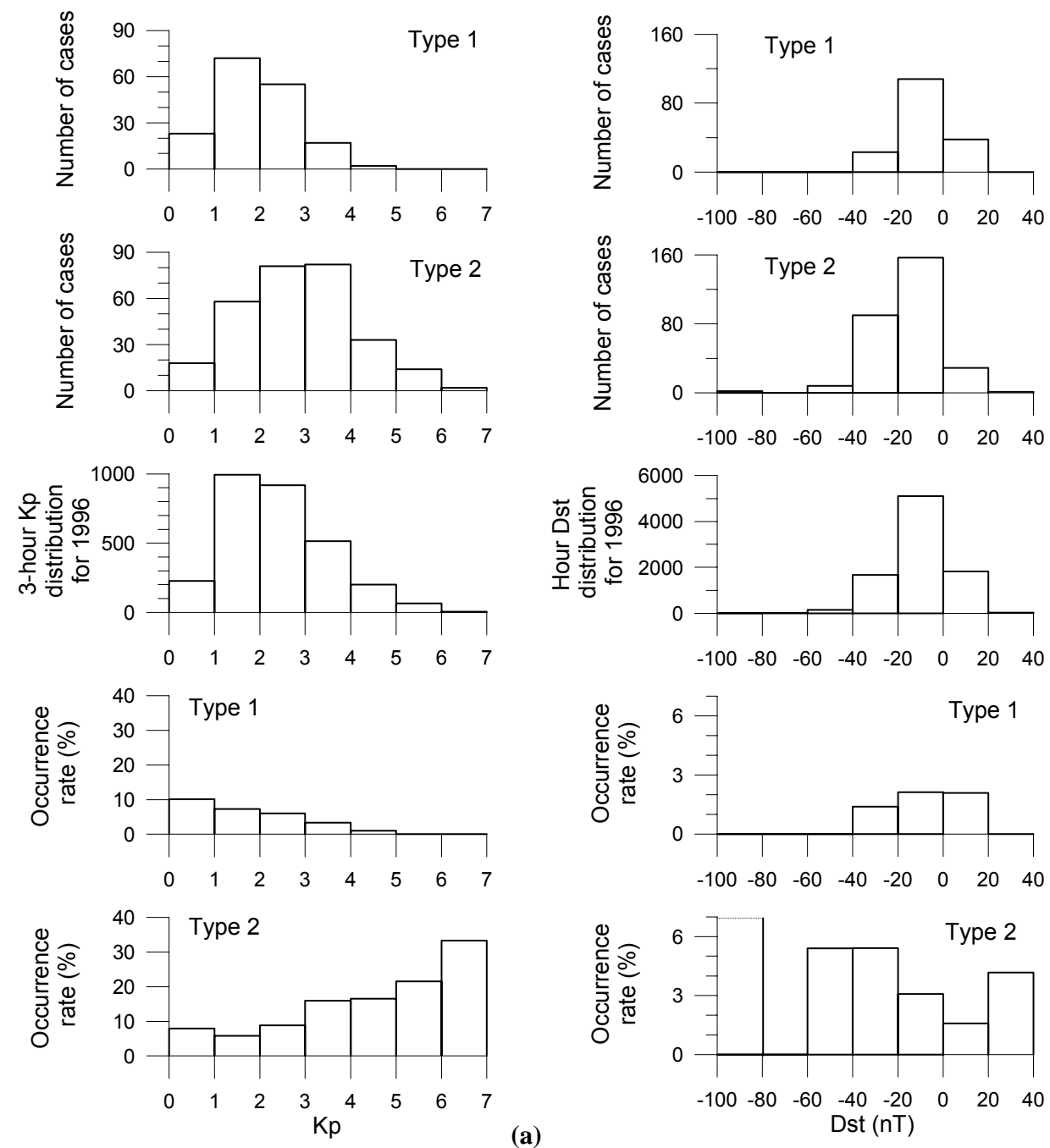

(b)

Fig. 4. Occurrence rate of localized proton precipitations as a function of geomagnetic activity indices $K_{p}$ (a) and $D_{s t}$ (b). From top to bottom: number of the Type 1 and 2 events as a function of the geomagnetic activity index, distribution of the index for 1996, occurrence rate of the Type 1 and 2 event observations as a function of the index.

sector. Neither LANL 1991-080 nor 1994-084 registered any particle injections (Fig. 6b). The lack of particle injection at geosynchronous orbit holds true for all Type 1 events for which the LANL data are available.

\subsection{Correspondence to the cold plasma at geosynchronous orbit}

The data from LANL spacecraft enable us to compare the localised proton precipitation with the behaviour of the cold plasmaspheric plasma at the geosynchronous orbit. Figures $5 \mathrm{c}$ and $6 \mathrm{c}$ present the data from the MPA instrument on board spacecraft 084 and 080 , respectively.

Figure $5 \mathrm{c}$ demonstrates a sharp enhancement of the cold plasma density in the equatorial plane in the region of 1418 MLT. The eastward edge of the enhancement is close to the meridian of the Type 2 event registration by the NOAA12 satellite. Figure $6 \mathrm{c}$ shows the MPA data for the event of
Type 1 . There were no significant cold plasma density variations during the event and around. Moreover, the density was very small (few particles per $\mathrm{cm}^{3}$ ); this means that the plasmasphere was well inside $6.6 R_{E}$ and there was no radial expansion of plasmaspheric plasma to the location of the energetic proton precipitation source.

The statistics of the cold plasma observations by the LANL spacecraft during the Type 1 and 2 events are shown in Fig. 7. The comparison was done for those cases when both the LANL spacecraft and the NOAA satellites were in the same MLT sector. The upper (bottom) panel presents the cold plasma density observed during the Type 1 (Type 2 ) events. The difference is clear: the Type 1 events occur when there is no cold plasma increase at the geosynchronous orbit. In contrast, the Type 2 events occur when cold plasma density varies up to some tens of particles per $\mathrm{cm}^{3}$. 


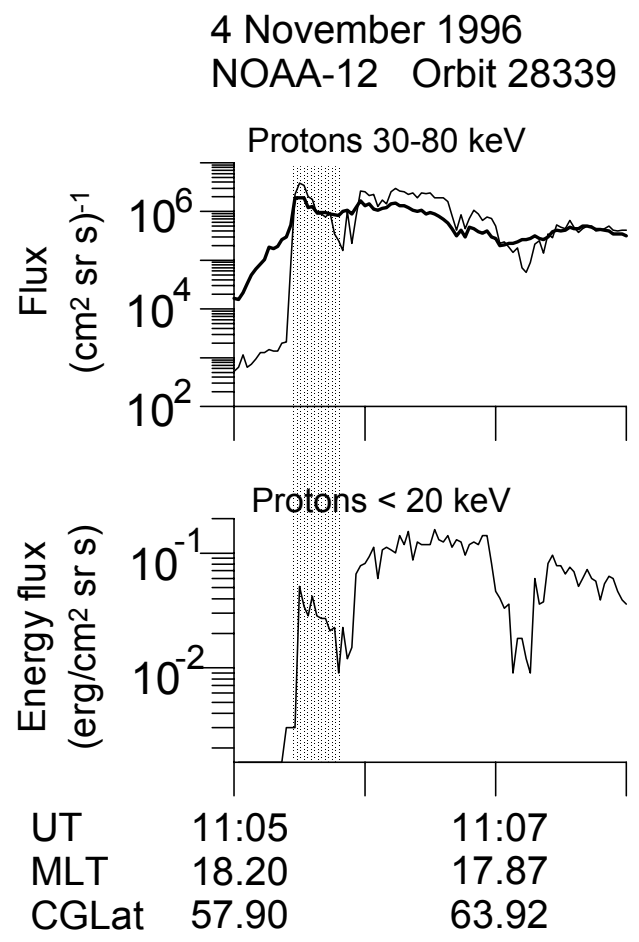

(a)

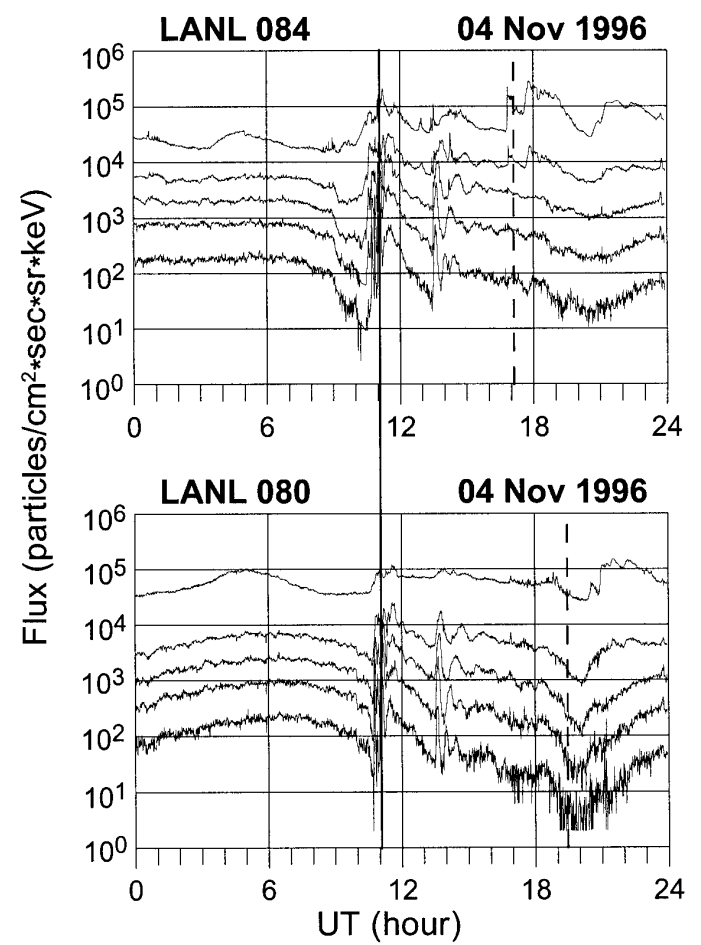

(b)
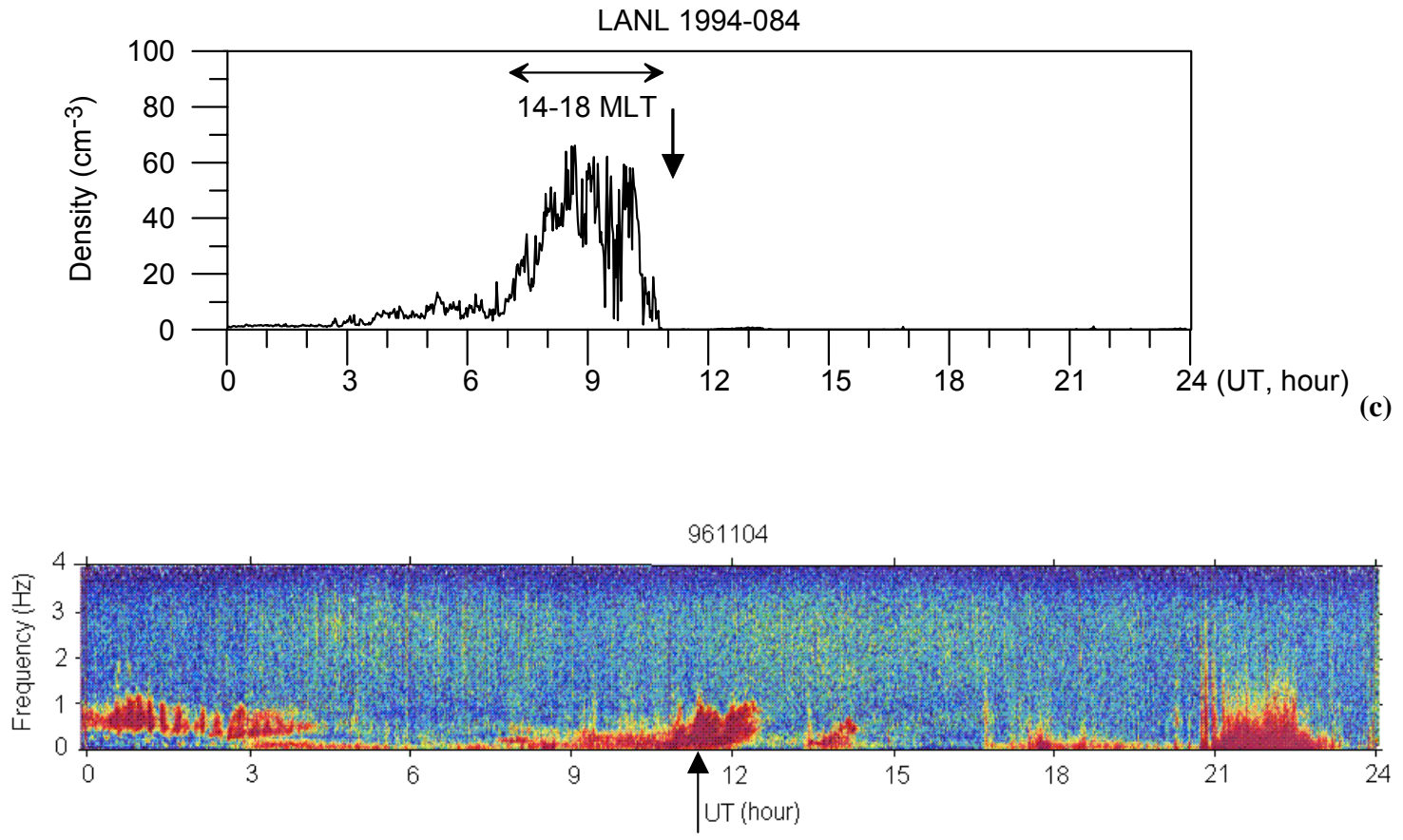

(d)

Fig. 5. The Type 2 event associated phenomena. (a) The NOAA-12 data showing the Type 2 event at 11:05:30 UT on 04 November 1996. (b) The SOPA instrument data showing the energetic proton fluxes on board the LANL spacecraft. The substorm injection is seen around 11:00 UT. Solid vertical line marks the time of the Type 2 event registration. On each panel a dashed vertical bar is plotted at the time for which the satellite was at local midnight. (c) The LANL MPA instrument data showing the cold plasmaspheric plasma increase in the 14-18 MLT range. (d) The daily spectrogram from SGO showing the dynamic spectra of geomagnetic variation in the range of $0.1-4 \mathrm{~Hz}$. Arrow marks the time of the NOAA-12 pass. The spectrogram shows the IPDP pulsations around the time of the NOAA-12 observation of the localized proton flux enhancement. 

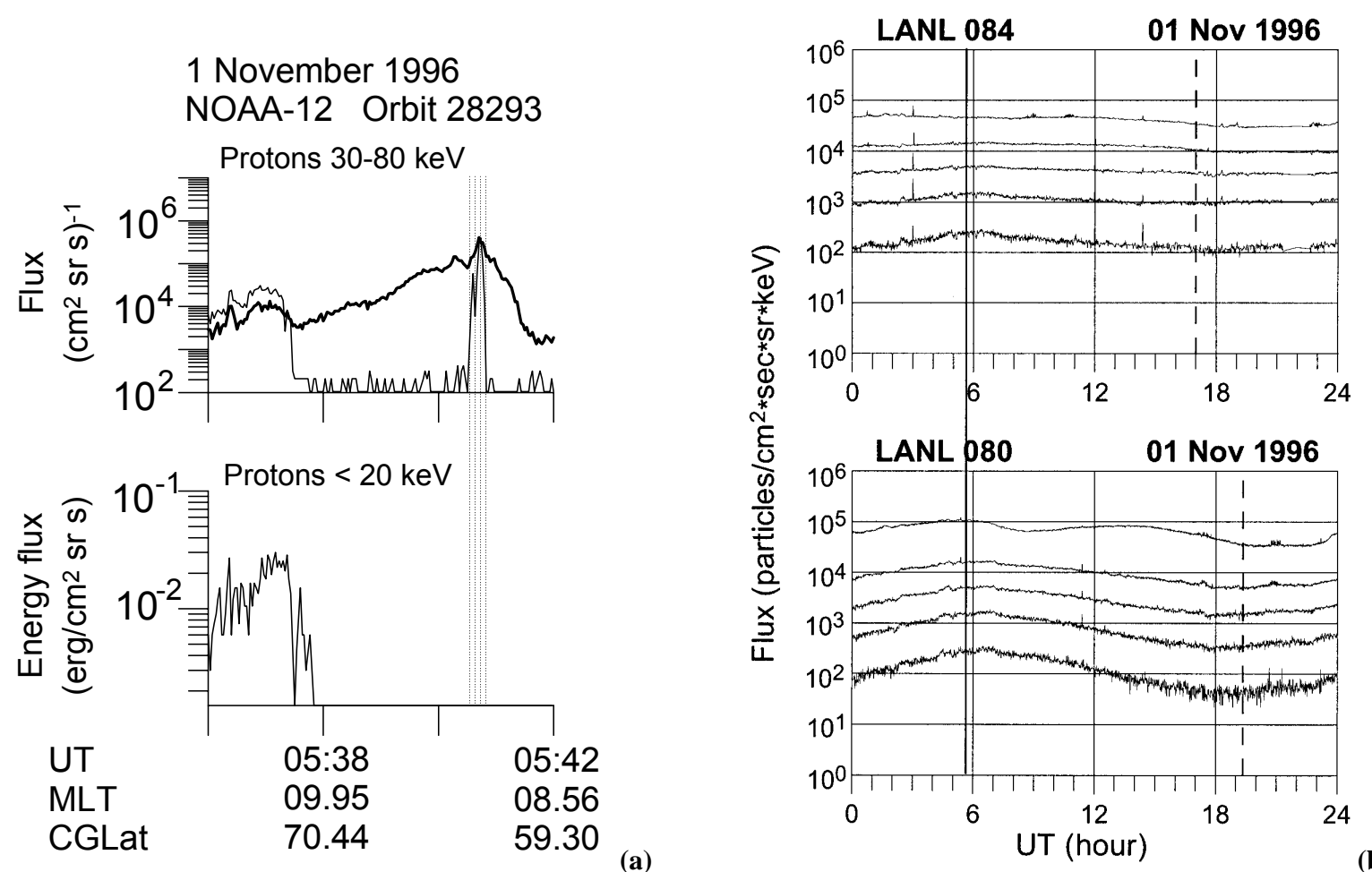

(a)

(b)

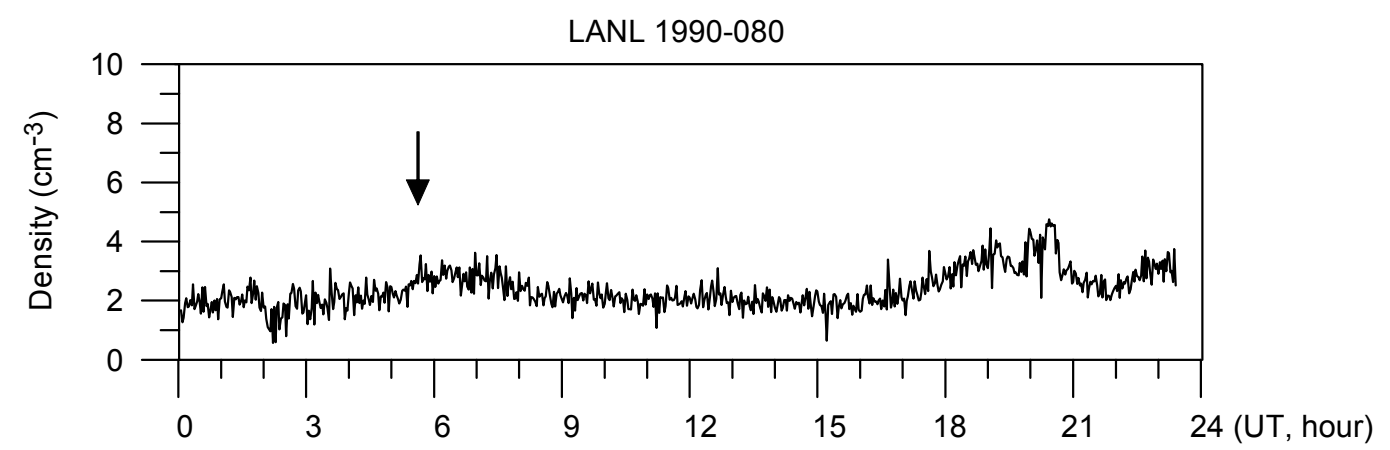

(c)

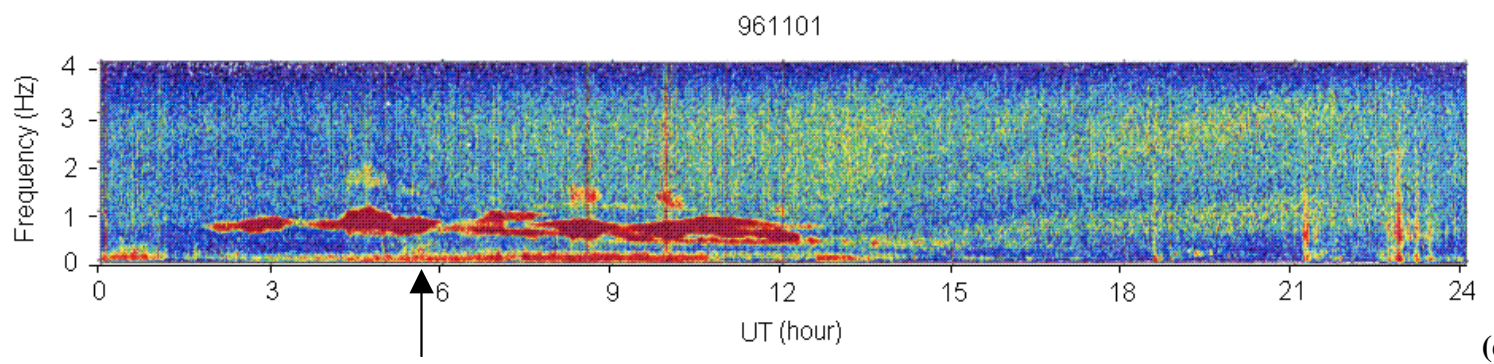

(d)

Fig. 6. The Type 1 event associated phenomena. (a) The NOAA-12 data showing the Type 1 event at 05:40:42 UT on 01 November 1996. (b) The SOPA instrument data showing the energetic proton fluxes on board the LANL spacecraft. Only smooth variations are seen. (c) The LANL MPA instrument data showing the lack of cold plasmaspheric plasma at geosynchronous distance. (d) The daily spectrogram from SGO showing the dynamic spectra of geomagnetic variation in the range of $0.1-4 \mathrm{~Hz}$. Arrow marks the time of the NOAA-12 pass. The Pc1 pulsations are seen on the ground during the localized proton flux enhancement. 


\subsection{Relation to Pc1 and IPDP}

To check if the localised energetic proton enhancements of Type 1 and 2 do relate to the EMIC waves, we considered ground-based records of geomagnetic pulsations in the Pc1 range made at SGO. Figures $5 \mathrm{~d}$ and $6 \mathrm{~d}$ show the daily spectrogram of geomagnetic fluctuation in the range of $0.1-4 \mathrm{~Hz}$. In each plot the arrow marks the time of the NOAA-12 pass shown in Figs. 5a and 6a. These figures demonstrate that during the localised proton precipitation, the ground station recorded geomagnetic pulsations. The pulsations were Pc1 during the Type 1 event and IPDP during the Type 2 event. To validate this relationship statistically, only those NOAA12 passes that occurred within $2 \mathrm{~h}$ of MLT of SGO were selected. The selection produced 35 cases corresponding to the Type 1 enhancements and 61 cases for the Type 2 events. In 33 cases (94\%), the Type 1 precipitation was accompanied by Pc1-2. For the remaining two cases we could not identify the pulsation type. Forty-six cases (75\%) of Type 2 events were accompanied by IPDP, 13 cases (22\%) were related to Pc1-2, and for two cases the type of pulsations was not identified.

\section{Summary}

We investigated two kinds of localised variations of energetic proton fluxes at low altitude within the anisotropic zone equatorward of the isotropy boundary. The common feature of these precipitation types is the presence of tens of $\mathrm{keV}$ precipitating protons measured by the MEPED instrument on board the NOAA satellite, but those events are distinguished either by the presence or absence of the low-energy component, as measured by the TED detector.

We found that the precipitation pattern containing the low energy component are more often isotropic and often accompanied by precipitation of high energy electrons. These precipitation events occur in the evening sector, under disturbed geomagnetic conditions, in association with energetic proton injections and significant increases in the cold plasma density at geosychronous orbit. The precipitation pattern is closely associated with IPDP.

The localised proton precipitation events without a lowenergy component are often anisotropic, and they are accompanied by the electron precipitation only in $44 \%$ of the cases. This precipitation occurs mostly in the morning-day sector, during quiet magnetic conditions, without substorm injections at geosynchronous orbit, and without any signatures of plasmaspheric plasma expansion to the geosynchronous distance. This precipitation pattern closely relates to Pc1.

\section{Discussion}

As mentioned in the Introduction, some features of the localised enhancements of the energetic proton flux at low altitudes have been studied by Lundblad and Søraas (1978), Søraas et al. (1999), Yahnina et al. (1998, 2000, 2002). These
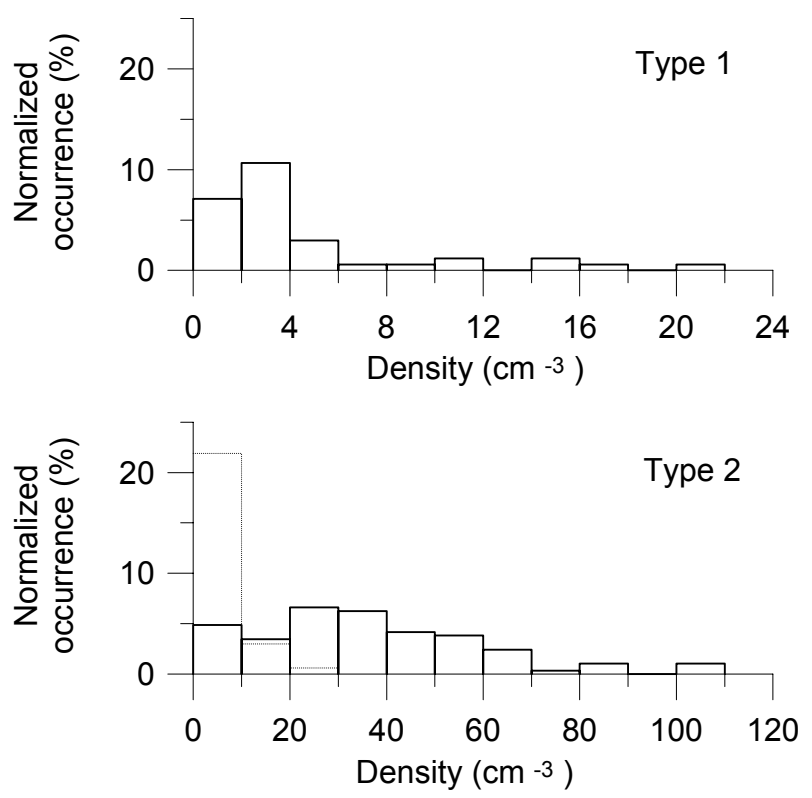

Fig. 7. Distribution of localised proton flux observations as a function of the cold plasma density at geosynchronous orbit. Upper panel: Normalised distribution of the Type 1 events. Bottom panel: Normalised distribution of the Type 2 events (solid line). For comparison the dashed line reproduces the Type 1 events distribution. Note the different scale on the plasma density axes in upper and bottom panels.

authors established the main morphological characteristics of the phenomenon, including the latitudinal width of the flux enhancements, their dependence on the geomagnetic storms, the MLT dependence, etc. Lunblad and Søraas (1978) and Søraas et al. (1999) noted the correlation of the enhancements (mainly in the locally trapped flux) with observations of the SAR arcs, while Yahnina et al. stressed the relationship between the localised energetic proton precipitation and structured Pc1 pulsations seen on the ground. Note that Yahnina et al. $(2000,2002)$ considered the precipitation pattern just the same as the Type 1 events described here (without precipitating low energy fluxes measured by the TED instrument, see, for example, Fig. 1 in Yahnina et al., 2000).

Søraas et al. (1999) concluded that localised enhancements of precipitating protons that were observed during the main phase of the geomagnetic storms occurred in the evening sector. The enhancements during the main phase were often associated with precipitation of energetic electrons. In contrast, the localised enhancements that were observed during the recovery phase tended to occur in the dayside and did not contain either energetic electrons or low-energy proton precipitation. This is in general agreement with the findings presented in this paper. Indeed, the Type 2 events, which have a maximum occurrence in the evening sector, are observed mainly during large negative $D_{s t}$, which is associated with the main or early recovery phase of a geomagnetic storm. The Type 1 events are observed during small $D_{s t}$; this corresponds to the late recovery 
phase or quiet geomagnetic conditions.

Søraas et al. (1980) described a few cases of localised energetic proton precipitation detected on board the ESRO1A satellite in the evening sector and related those events to IPDPs observed on the ground. Our statistics of the Type 2 events is in agreement with their findings.

Our study clearly indicates that precipitation of Types 1 and 2 are, respectively, counterparts of the Pc1 and IPDP pulsations observed on the ground. Thus, the precipitation of both types manifests the operation of the ion-cyclotron instability (but in different magnetospheric conditions) and marks the field lines of the wave-particle interaction source region.

Bräysy et al. (1998) considered EMIC waves during geomagnetic storms using the low-altitude Freja satellite data. These authors found that during the main phase of the geomagnetic storm the EMIC waves were more intense and they were located in the evening MLT sector, in contrast to the pre-storm and recovery phases, when the amplitudes of the EMIC waves were smaller and the waves were observed in the morning-day sectors. These findings are in agreement with the results by Erlandson and Ukhorskiy (2001), who investigated the EMIC waves observed by the DE-1 satellite near to the equatorial plane. They found statistically that more intense EMIC waves occur in the evening sector. One may expect that more intense waves scatter the particles more effectively and produce more intense and isotropic precipitation. This agrees very well with our division of the localised proton enhancements into those related to Pc1 and IPDP. The IPDP related proton fluxes are more intense in comparison with those related to Pc1; they are more isotropic and their energy spectra are wider. Finally, the IPDP related proton precipitation does occur in the evening MLT sector during high levels of geomagnetic activity. Thus, their morphology is similar to that established for the intense EMIC waves. The same holds true for the correlation of the precipitation related to Pc1 and less intense EMIC waves observed at the recovery phase.

Bräysy et al. (1998) noted that intense EMIC waves during the main phase of the storm did not correlate with groundbased Pc1 pulsations. Perhaps, this apparent contradiction might be explained by our findings which show that in the evening sector, during geomagnetic disturbances, the EMIC waves are associated with IPDP, and not Pc1. Weaker EMIC waves during the recovery phase in the morning-day sector are detected on the ground as Pc1. Note that, because of the localised nature of the source and the fast motion of the satellite across the source field lines, the EMIC waves detected by the low-altitude satellites have a small duration (e.g. Iyemori and Hayashi, 1989; Mursula et al., 1994; Erlandson and Anderson, 1996; Bräysy et al., 1998). Thus, it is hard to distinguish between IPDP and Pc1 from the space (low-altitude) wave measurements.

The probability of observing Pc1/IPDP related precipitation is small ( $\sim 1 \%$ and $2 \%$ ), respectively. Note that Erlandson and Ukhorskiy (2001) found that the EMIC waves have an occurrence rate of the same order, and it is higher during storm time than during quiet conditions. This agrees with the fact that IPDPs are observed during disturbances, and Pc1s are mainly observed during geomagnetically quiet periods.

The difference between the Type 1 and 2 precipitation energy spectra is also explained by their different relationship to the geomagnetic disturbances. IPDP are observed during particle injections, i.e. when the density of energetic protons is higher and the mean energy is lower. Typical mean energies of quiet-time ring current protons is about $100 \mathrm{keV}$, while during the main phase it can decrease to about 20$30 \mathrm{keV}$ (e.g. Hamilton et al., 1988). This enables the participation of lower-energy protons in the wave generation and pitch-angle diffusion. Higher-energy particles can be influenced by waves excited by lower-energy ones, since they satisfy the resonance condition $\omega-\Omega_{H}=k v_{\|}$, with the same waves at some distance from the equatorial generation region, i.e. at higher geomagnetic latitudes (e.g. Bespalov and Trakhtengerts, 1986). This influence increases with the wave energy density, which is directly related to the number density of ring-current ions. Therefore, the energy range of particles involved in the interaction increases as their number density increases. During a recovery phase, the characteristic energy of protons generating the waves increases, and their number density decreases. Consequently, the emissions are weaker and are generated in a narrower frequency band, resonant only with the dominant component of proton energy.

Keeping in mind the duration of the ground $\mathrm{Pc} 1$ pulsations, one may conclude that the Pc1 source typically operates for several hours. In such case, the small probability of observing Pc1 related precipitation at low altitudes means that the Pc1 source is longitudinally confined (see also the discussion in Yahnina et al., 2000, 2002). The duration of the IPDP events is relatively small (few tens of minutes). This may also be the reason for the low occurrence rate $(\sim 2 \%)$ of the related precipitation events. The localised nature of the EMIC wave source at the boundary of the cold plasmaspheric plasma follows, not only from the observations, but also from the self-consistent consideration of the interaction of the ring current ions and cyclotron waves (Trakhtengerts et al., 1997).

The details of the Pc1 and IPDP dynamic spectra formation are still unclear (see, Kangas et al., 1998 for a comprehensive discussion and references). However, we believe that the difference between the spectra of these two types of the EMIC waves is directly related to different conditions in the source region. Our data show that the conditions during the IPDP-related precipitation are non-stationary. These data imply (Fig. 5) that the IPDP source operates when and where the newly injected and westward-drifting hot proton cloud meets a boundary of the dense plasmaspheric plasma (see, also Horita et al., 1979; Søraas et al., 1980). This happens in the evening sector in the region of the plasmaspheric bulge or detached tail-like structure, as evidenced by the MPA data. The duration of the IPDP event can be related to the longitudinal size and structure of the injected proton cloud. The presence of the low energy component in the proton precipitation may relate to the enhanced cold plasma density. The Pc1 generation seems to be a quasi-steady process. It sug- 
gests the existence of a long-lived cold plasma inhomogeneity (a cold plasma cloud or tube) within the geosynchronous orbit, which is in permanent interaction with the ring current population. It is quite possible that there exists several such inhomogeneities (Pc1 sources); in such case one may expect multi-band Pc1 on the ground (see discussion in Yahnina et al., 2000).

Note that, besides the magnetospheric source region, the ionosphere can also play an important role in the formation of the dynamic spectra of ULF $0.1-5 \mathrm{~Hz}$ waves, due to their frequencies being close to the eigenfrequencies of the ionospheric Alfvén resonator (IAR; see Belyaev et al., 1984; Trakhtengerts et al., 2000a). Kleimenova et al. (1995) concluded that the IPDP pulsations typically coincide in latitude with the position of the main ionospheric trough. On this basis they supported the idea that the wide spectrum typical for IPDP is formed under disturbed conditions, since the IAR influence is insignificant (i.e. the IAR quality is low and/or the eigenfrequencies are higher than the pulsation frequency). On the contrary, Pc1 pearls are formed where the reflecting ionosphere forms a good Alfvén resonator, which is more typical for quiet conditions. Such arguments were used also by Trakhtengerts et al. (2000b), to explain why Pc1 pearls and spectral resonance structures in magnetic background noise exhibit the same anticorrelation with solar activity.

The fact that the presence of energetic electrons is associated with proton precipitation is puzzling. In contrast to Søraas et al. (1999), who found the enhancement of the $>300 \mathrm{keV}$ electrons associated with proton precipitation bursts only during the main phase of a geomagnetic storm, we detected the electron precipitations at energies $>30 \mathrm{keV}$ in $44 \%$ of the Type 1 events (occurring mainly during the late recovery phase and quiet conditions) and in more than $80 \%$ of the Type 2 events, which occur during early recovery. It was suggested (Thorne and Kennel, 1971) that ion cyclotron waves can precipitate the relativistic electrons $(E \approx 1 \mathrm{MeV})$, but this theory is hardly applicable to the 30 $\mathrm{keV}$ particles. Nevertheless, the fact that proton and electron precipitation may be coincident explains the experimental result by Pikkarainen et al. (1986), who found the correlation between the IPDP events and riometer absorption, which is evidently due to energetic electron precipitation. We suggest that energetic electrons may be precipitated due to the cyclotron resonant interactions with whistler waves in the ELF/VLF range, excited at and guided by the same coldplasma inhomogeneities as the EMIC waves that scatter the ring current protons.

\section{Conclusion}

We found specific precipitation patterns which seem to be the particle counterparts of geomagnetic pulsations Pc1 and IPDP. These patterns exhibit a different morphology, which correlates very well with the known features of the Pc1 and IPDP pulsations observed from the ground and with the mor- phology of the EMIC waves observed in space. We showed that the two precipitation patterns appear under different magnetospheric conditions, and this can be the reason for their morphological differences.

The finding that the precipitation patterns are related to the ion-cyclotron waves fills the long-existent gap in the study of the interaction of the ring current population and ioncyclotron waves. The characteristics of the precipitation can be useful for a better understanding of the magnetosphereionosphere processes, including the losses of the ring current particles and the ionosphere modification induced by the precipitation. The later may play a role in the formation of bouncing Alfvén wave packets (Belyaev et al., 1984).

Acknowledgements. The work was supported by INTAS (grant 990335) and by Russian Foundation for Basic Research (grant 01-0564437). The Russian co-authors acknowledge the support from the Division of Physical Sciences of the Russian Academy of Sciences (program DPS-16). The NOAA POES program is supported by the National Oceanic and Atmospheric Administration and the National Environmental Satellite, Data, and Information Service.

Topical Editor T. Pulkkinen thanks R. Erlandson and N. Kleimenova for their help in evaluating this paper.

\section{References}

Bame, S. J., McComas, D. J., Thomsen, M. F., Barraclough, B. L., Elphic, R. C., Glore, J. P., Gosling, J. T., Chavez, J. C., Evans, E. P., and Wymer, F. J.: Magnetospheric Plasma Analyzer for Spacecraft with Constrained Resources, Rev. Sci. Instr., 64, 1026, 1993.

Belian, R. D., Gisler, G. R., Cayton, T., and Christensen, R.: HighZ Energetic Particles at Geostationary Orbit During the Great Solar Proton Event Series of October 1989, J. Geophys. Res., 97, 16897, 1992.

Belyaev, P. P., Polyakov, S. V., Rapoport, V. O., and Trakhtengerts, V. Yu.: On the fine structure of the Alfvén maser radiation, Geomagn. Aeron., 24, 2, 242-248, 1984.

Bespalov, P. A. and Trakhtengerts, V. Yu.: The cyclotron instability in the Earth radiation belts, in: Reviews of Plasma Physics, edited by Leontovich, M. A., Plenum, N.Y., 10, 155-192, 1986.

Bespalov, P. A., Demekhov, A. G., Grafe, A., and Trakhtengerts, V. Yu.: On the role of collective interaction in asymmetric ring current formation, Ann. Geophysicae, 12, 422-430, 1994.

Bräysy T., Mursula, K., and Marklund, G.: Ion cyclotron waves during a great magnetic storm observed by Freja double-probe electric field instrument, J. Geophys. Res., 103, 4145-4155, 1998.

Christon, S. P., Mitchell, D. G., Williams, D. J., Huang, C. Y., Frank, L. A., and Eastman, T. E.: Energy spectra of plasma sheet ions and electrons from $\sim 50 \mathrm{eV} / \mathrm{e}$ to $\sim 1 \mathrm{MeV}$ during plasma sheet temperature transitions, J. Gephys. Res., 93, 2562-2572, 1988.

Christon, S. P., Williams, D. J., Mitchell, D. G., Frank, L. A., and Huang, C. Y.: Spectral characteristics of plasma sheet ion and electron populations during undisturbed geomagnetic conditions, J. Geophys. Res., 94, 13 409-13 424, 1989.

Cornwall, J. M.: Cyclotron instabilities and electromagnetic emission in the ultra low frequency ranges, J. Geophys. Res., 70, 6170,1965 
Erlandson, R. E. and Anderson, B. J.: Pc1 waves in the ionosphere: A statistical study, J. Geophys. Res., 101, 7843-7857, 1996.

Erlandson, R. E. and Ukhorskiy, A. J.: Observations of electromagnetic ion cyclotron waves during geomagnetic storms: Wave occurrence and pitch angle scattering, J. Geophys. Res., 106, $3883-$ 3895, 2001.

Gintzburg, M. A.: On the new mechanism of excitation of the Earth's magnetic field micropulsation, Izv. AN SSSR, Ser. Geophys., 11, 1679-1691, (in Russian), 1961.

Grafe, A., Trakhtengerts, V. Yu., Bespalov, P. A., and Demekhov, A. G.: Evolution of the low latitude geomagnetic storm field and the importance of turbulent diffusion for ring current particle losses, J. Geophys. Res., 101, 11, 24 689-24 706, 1996.

Gvozdevsky, B. B. and Sergeev, V. A.: Scattering on the current sheet as a possible mechanism of precipitation of the auroral protons, Geomagn. Aeron., 35, 4, 151-155, 1995.

Hamilton, D. C., Gloeckler, G., Ipavich, F. M., Studemann, W., Wilken, B., and Kremser, G.: Ring current development during the great geomagnetic storm of February 1986, J. Geophys. Res., 93, 14 343-14 355, 1988.

Hayakawa, M., Shimakura, S., Koboyashi, T., and Sato, N.: A study of polarisation of irregular pulsations of diminishing period and their generation mechanism, Planet. Space Sci., 40, 1081-1091, 1992.

Heacock, R. R. and Kivinen, M.: Relation of Pc1 micropulsations to the ring current and geomagnetic storms, J. Geophys. Res., 77, 6746-6760, 1972.

Hill, V. D., Evans, D. S., and Sauer, H. H.: TIROS/NOAA satellites space environment monitor. Archive tape documentation, NOAA Tech. Mem. ERL SEL-71, 50 pp. Environs. Res. Lab., Boulder. 1985.

Horita, R. E., Barfield, J. N., Heacock, R. R., and Kangas, J.: IPDP source regions and resonant proton energies, J. Atmos. Terr. Phys., 41, 293-309, 1979.

Iyemori, T. and Hayashi, K.: Pc1 micropulsations observed by Magsat in the ionospheric F-region, J. Geophys. Res., 94, 93100, 1989.

Kangas, J., Guglielmi, A., and Pokhotelov, O.: Morphology and physics of short-period magnetic pulsations (a review), Space Science Reviews, 83, 435-512, 1998.

Kleimenova, N. G., Kangas, J., Pikkarainen, T., and Ranta, K.: Geomagnetic IPDP pulsations and the major ionospheric trough, Geomagn. Aeron., 35, 6, 60-68, 1995.

Kozyra, J. U., Jordanova, V. K., Horne, R. B., and Thorne, R. M.: Modeling of the contribution of electromagnetic ion cyclotron (EMIC) waves to stormtime ring current erosion, AGU monograph on magnetic storms, in: Magnetic Storms, Geophysical Monograph 98, AGU, Washington D.C., 187-202, 1997.

Kroehl, H. W.: Space environment monitoring by low-altitude satellites, in: The IMS source book. Guide to the international magnetospheric study data analysis, edited by Russel, C. T. and Southwood, D. J., AGU, Washington D.C., 99-105, 1982.

Lundblad, J. A. and Søraas, F.: Proton observations supporting ion cyclotron wave heating theory of SAR arc formation, Planet. Space Sci., 26, 245-254, 1978.

McComas, D. J., Bame, S. J., Barraclough, B. L., Donart, J. R.,
Elphic, R. C., Gosling, J. T., Moldwin, M. B., Moore, K. R., and Thomsen, M. F.: Magnetospheric plasma analyzer: initial threespacecraft observations from geosynchronous orbit, J. Geophys. Res., 98, 13 453-13 465, 1993.

Mursula, K., Blomberg, L. G., Lindquist, P.-A., Marklund, G. T., Bräysy, T., Rasinkangas, R., and Tanskanen, P.: Dispersive Pc1 bursts observed by Freja, Geophys. Res. Lett., 21, 1851-1854, 1994.

Newell, P. T., Sergeev, V. A., Bikkuzina, G. R., and Wing, S.: Characterizing the state of the magnetosphere: testing the ion precipitation maxima latitude (b2i) and the ion isotropy boundary, J. Geophys. Res., 103, 4739-4745, 1998.

Pikkarainen T., Kangas, J., Ranta, H., Ranta, A., Maltseva, N., Troitskaya, V., and Afanasieva, L.: Riometer absorption events in the evening-to-afternoon sector of the auroral and sub-auroral zone and movements of the IPDP source, J. Atmos. Terr. Phys., 48, 585-596, 1986.

Søraas, F., Lundblad, J. A., Maltseva, N. F., Troitskaya, V., and Selivanov, V.: A comparison between simultaneous IPDP groundbased observations of energetic protons obtained by satellites, Planet. Space Sci., 28, 387-405, 1980.

Søraas, F., Aarsnes, K., Lundblad, J. A., and Evans, D. S.: Enhanced pitch angle scattering of protons at mid-latitudes during geomagnetic storms, Phys. Chem. Earth (C), 24, 287-292, 1999.

Thorne, R. M. and Kennel, C. F.: Relativistic electron precipitation during magnetic storm main phase, J. Geophys. Res., 76, 44464453, 1971.

Trakhtengerts, V. Yu., Demekhov, A. G., and Grafe, A.: Threedimensional magnetospheric currents due to energetic particle precipitation, Geomagn. Aeron., 37, 4, 9-16, 1997.

Trakhtengerts, V. Y., Demekhov, A. G., Polyakov, S. V., Belyaev, P. P., and Rapoport, V. O.: A mechanism of Pc1 pearl formation based on the Alfvén sweep maser, J. Atmos. Solar-terr. Phys., 62, 231-238, 2000a.

Trakhtengerts, V. Y., Demekhov, A. G., Belyaev, P. P., Polyakov, S. V., Ermakova, E. N., and Isaev, S. V.: A mechanism of anticorrelation in occurrence of ULF electromagnetic noise resonance structure and Pc1 observations with the solar activity cycle, J. Atmos. Solar-terr. Phys., 62, 253-256, 2000 b.

Troitskaya, V. A.: Pulsations of the Earth's electromagnetic field with periods of 1 to 15 seconds and their connection with phenomena in high atmosphere, J. Geophys. Res., 66, 5-18, 1961.

Wentworth, R. C.: Enhancement of hydromagnetic emissions after geomagnetic storms, J. Geophys. Res., 69, 2292-2298, 1964.

Yahnina, T. A., Titova, E. E., and Yahnin, A. G.: Localised precipitation of energetic protons at subauroral latitudes, in "Physics of auroral phenomena”, Proc. XXI Annual Seminar, Apatity, 113116, 1998.

Yahnina, T. A., Yahnin, A. G., Kangas, J., and Manninen, J.: Proton precipitation related to Pc1 pulsations, Geoph. Res. Lett., 27, 3575-3578, 2000.

Yahnina, T. A., Yahnin, A. G., Kangas, J., and Manninen, J.: Localized enhancements of energetic proton fluxes at low altitudes in the subauroral region and their relation to the Pc1 pulsations, Cosmic Research, N 3, 213-223, 2002. 